\title{
Comparing discourse tree structures
}

\author{
Elena Mitocariu ${ }^{1}$, Daniel-Alexandru Anechitei ${ }^{1}$, Dan Cristea ${ }^{1,2}$ \\ 1 "Al.I.Cuza" University of Iasi, Faculty of Computer Science \\ 16, General Berthelot St., 700483 - Iasi, Romania \\ ${ }^{2}$ Romanian Academy, Institute for Computer Science \\ 2, T. Codrescu St., 700481 - Iasi Romania \\ \{elena.mitocariu, daniel.anechitei, dcristea\}@info.uaic.ro
}

\begin{abstract}
The existing discourse parsing systems make use of different theories to put at the basis of processes of building discourse trees. Many of them use Recall, Precision and F-measure to compare discourse tree structures. These measures can be used only on topologically identical structures. However, there are known cases when two different tree structures of the same text can express the same discourse interpretation, or something very similar. In these cases Precision, Recall and F-measures are not so conclusive. In this paper, we propose three new scores for comparing discourse trees. These scores take into consideration more and more constraints. As basic elements of building the discourse structure we use those embraced by two discourse theories: Rhetorical Structure Theory (RST) and Veins Theory, both using binary trees augmented with nuclearity notation. We will ignore the second notation used in RST - the name of relations. The first score takes into account the coverage of inner nodes. The second score complements the first score with the nuclearity of the relation. The third score computes Precisions, Recall and F-measures on the vein expressions of the elementary discourse units. We show that these measures reveal comparable scores there where the differences in structure are not doubled by differences in interpretation.
\end{abstract}

Keywords: discourse parser, Rhetorical Structure Theory, Veins Theory, evaluation, discourse tree structure

\section{$1 \quad$ Introduction}

Discourse parsing systems have an important role in many NLP applications such as question-answering, summarization or deep understanding of texts. Driven by one or another of the discourse theories, the majority of parsers produce tree structures. However, although correctly revealing relations between parts of the discourse, the topology of trees sometimes evidence differences which are not anchored in true discourse facts. To give just one example, a narration (sequence of elementary discourse units of equal salience) could be represented by binary trees in many ways. This paper proposes new measures of evaluating discourse trees which shadows idiosyncrasies of 
notation, while bringing forth differences which are supported by discourse phenomena.

We propose three scores for comparing the discourse trees and we exemplify them from the point of view of Rhetorical Structure Theory and Veins Theory. The first score takes into account only the coverage of the nodes. The second score takes into consideration the nuclearity of the relations. The third score computes Precisions, Recall and F-measures on vein expressions. These metrics can be applied to either RST or VT annotated trees.

Among the theories dealing with the organization of the discourse, Rhetorical Structure Theory (RST) is well accepted and, when the discourse structure should be seen in correlation with referentiality, Veins Theory (VT) proposes to look at the discourse structure from another angle. Both use binary tree ${ }^{1}$ representations, in which leafs are elementary discourse units (edus), such as clauses or short sentences, and internal nodes represent larger text spans. RST uses a labeling function that attaches relation names and nuclearities to its inner nodes, while VT ignores the names of relations. Because of this simplified representation, one can say that VT is included in RST. For instance, on an annotated RST corpus one can calculate head the vein expressions, which are of primary importance only in VT. However, the conclusions that VT develops go in a different direction then those set by RST.

To compare discourse structures, the PARSEVAL measures [1], based on Precision, Recall and F-measure, are used. The main discontent is that these measures cannot be used to account for structures which, although different, have rather similar interpretations.

In the following, we will consider binary discourse trees on which parent nodes specify the nuclearities of the left and right daughters. For example, in Fig. 1 the node labeled 1 is a nucleus and the node labeled 2 is a satellite.

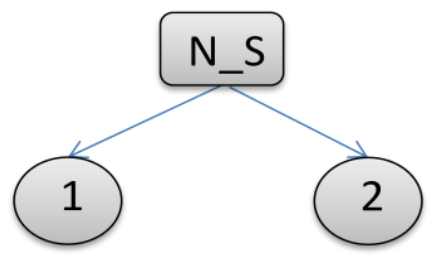

Fig. 1. The discourse tree representation

The automatic identification of discourse structure should include, as a preliminary step, a method for the segmentation of the discourse in $e d u$ s. Discourse tree structures are the result of discourse parsing systems and many of them are developed based on the RST terminology. Since Marcu's first attempt at developing a rule-based dis-

\footnotetext{
${ }^{1}$ A binary tree is a tree in which every node has at most two children. The binary trees used to represent discourse structures are always strict (proper) binary trees (each parent node has exactly two children).
} 
course parser [2] on the base of discourse markers, several algorithms for discourse parsing have been proposed, both statistical and rule-based [3]. The SPADE algorithm doesn't use cue words for identification of rhetorical relations [4], using instead syntactic and lexical fetures. It is a sentence-level discourse parser which exploits a set of lexico-gramatical dominance relations corresponding to the syntactic representation of sentences. In [5] is described an algorithm that scores and labels relations between spans, where the discourse parse trees are the result of a supervised leaning. A probabilistic parser for parsing the discourse structure of dialogue is presented in [6].

In the following, we make first a brief presentation of the two theories we have used to exemplify the structure of discourse: Rhetorical Structure Theory - in section 2 , and Veins Theory - in section 3, by insisting only on notions linked to representational issues. In section 4 we introduce and discuss the scores and in section 5 we give some conclusions.

\section{Rhetorical Structure Theory}

Rhetorical Structure Theory developed by Thompson and Mann is one of the most used theories among those dealing with the structure of discourse. It is used in discourse analysis and text generation [7]. Like in the majority of discourse theories, RST segments the text in $e d u$ s, which could be clauses or sentences expressing elementary events, situations, statements. In RST, the spans of the text have a different importance in the whole discourse. Nuclei are the most important spans in the text and are compulsory for the coherence of the discourse. When they are edus, their deletion makes the text incoherent. When they are larger than edus, at least parts of them should be kept if a simplification is intended. Unlike nuclei, satellites only give additional information. They complement the statements/situations/events communicated by the nuclei and, if deleted from the text, the overall understability will not be harmed, even if some details will be missing.

Thus, the discourse includes nucleus-satellite relations and multinuclearity relations [8]. In a nucleus-satellite (hypotactic) relation, the whole span includes exactly one nucleus and any number of satellites. In a multinuclear (paratactic) relation, all subspans have the same importance and are considered nuclear. Although, the RST trees are not necessarily binary, equivalent transformations can be imagined that make them binary. For instance, a 3-arguments paratactic relation can be transformed into 2 binary paratactic relations with the same name.

\section{Veins Theory}

Taking from RST the notion of nucleus and satellite, but ignoring the name of the relations, VT attaches two expressions to each node of a binary discourse tree: the head and the vein.

The head expression is a list of the most salient $e d u$ s that the node covers and is calculated bottom-up, as follows: 
- if the node is a leaf, the head expression is its label;

- else, the head expression is the concatenation of the head expressions of its nuclear children.

By definition, the vein expression of a node $n$ is that list of elementary discourse units which are of primary importance for understanding the span the node $n$ covers in the context of the whole discourse. It is calculated top-down, as follows:

- the vein expression of the root node is its head expression;

- otherwise, if the node is a nucleus and his father has the vein expression $v$, then:

- if the node has a left satellite sibling with head $h$, then its vein expression is $\operatorname{seq}(\operatorname{mark}(h), v)$;

- else: $v$;

- finally, if the node is a satellite with the head $h$ and the vein of his father is $v$, then:

- if it is a left son, its vein expression is seq $(h, v)$;

- else its vein expression is $(\operatorname{seq}(h, \operatorname{simpl}(v))$.

The seq, simpl, mark, are function which can be described thus:

- seq returns the concatenation of two sequences of nodes, considering the frontier ordering, from left to right;

- mark returns the same symbols as in its argument, but marked in some way (for example between parentheses or primed);

- simpl eliminates all marked symbols from its argument [9].

\section{The Scores}

In discourse representation theories, it is notorious that a certain vision on a discourse tree structure can be mirrored by different tree topologies, which not necessarily express significant differences in the interpretation. As such, because representing discourse structures by trees has become a standard in many theories (SDRT, RST, VT, etc.), it would be useful to have a measure by which to compare these structures and show to what extend they mean the same thing or not. In the technology of discourse parsing, this issue is particularly important for at least three reasons. First, a corpus of gold files should be prepared by hand, using the competencies of human annotators. And, because the appreciation of a discourse structure is to such a large extend subjective, we would like to know the opinions of more experts on the issue. Then, whenever corpora are built in NLP and more annotators are involved, their outcomes should be compared for agreement. Second, evaluation of a discourse parser is usually done by comparing the parser's output against this gold representation contributed by humans. Finally, modifying certain parameters of the parser could yield trees which are slightly different, while most of the rest of the structure could be shared. But how much identical are these trees? Where in the structure of the tree, differences start to 
be significant? To what extend some differences can be ignored, therefore characterizing stable decisions of the parser?

In this section we will propose a number of scores that are intended to offer better comparisons between discourse tree structures.

There are many possibilities to represent by binary trees a discourse structure. The number of different binary trees that can be drawn with $n$ terminal nodes is given by the Catalan numbers [10]:

$$
\# \text { trees }=\frac{(2 * n-2) !}{n !(n-1) !} .
$$

For example, in case of a discourse with 4 edus, formula (1) above indicates 5 different binary trees, which are depicted in (Fig. 2).

\subsection{The Overlapping Score}

In [3] it is shown that a discourse tree can be formally represented as a set of tuples of the form $\mathrm{R}_{\mathrm{k}}[i, m, j]$, denoting a relation and its two arguments, as text spans: the left one spanning between $e d u$ s $i$ and $m$, and the right one spanning between $e d u$ s $m+1$ and $j$. For example, in the tree of Fig. 2 (c) the tuples are : $\mathbf{R}_{1}[1,1,4], \mathbf{R}_{2}[2,2,4]$ and $\mathrm{R}_{3}[3,3,4]$. The tuple representing the root relation will cover all leafs.

The Overlapping Score (OS) is taking into consideration only the coverage of the nodes. The representation with tuples can be used for that. By enduring an abuse of language, we will say that two tuples overlap if their starting and ending positions are identical $\left(i_{1}=i_{2}\right.$ and $\left.j_{1}=j_{2}\right)$. With this convention, the score will be computed by dividing the number of overlapping tuples of a discourse tree structure of the same text, to the total numbers of tuples (number of leafs -1) as presented in equation (2):

$$
\text { OS }=\frac{\text { \#overlapping tuples }}{\text { \#total tuples }} .
$$

At least one pair of tuples are overlapping, the ones describing the root relation and which should cover the whole range of nodes. Two different tree structures with the same text segmentation in clauses will have the same number of leafs.

To exemplify OS, we will compute it for some pairs of trees in the family of trees of (Fig. 2) (imagining that one represents a gold file and the second is contributed by a discourse parser). For example if we consider the discourse trees from Fig. 2(a) and Fig. 2(b), the number of overlapping tuples is two: $R_{3}[1,1,2]$ overlaps with $R_{2}[1,1,2]$ (covering nodes: 1 and 2) and $R_{1}[1,3,4]$ is identical with $R_{1}[1,2,4]$ (covering nodes 1 , $2,3,4)$. The total number of tuples for this example is 3 . The remaining pair of tuples $\left(\mathrm{R}_{2}[1,2,3]\right.$ and $\mathrm{R}_{3}[3,3,4]$ are not overlapping because $i_{1} \neq i_{2}$ and also $j_{1} \neq j_{2}$ Applying equation (2) we obtain $\mathrm{OS}=2 / 3$.

If we analyze the trees from Fig. 2(a) and Fig. 2(c), the number of overlapping tuples is 1 and the OS will be 1/3. Thus we can conclude that the discourse tree structure from Fig. 2(a) is more similar to the discourse tree structure of Fig. 2(b) than to 
that in Fig. 2(c). If OS equals 1 it means that the two discourse trees structures are identical from the point of view of the topology.

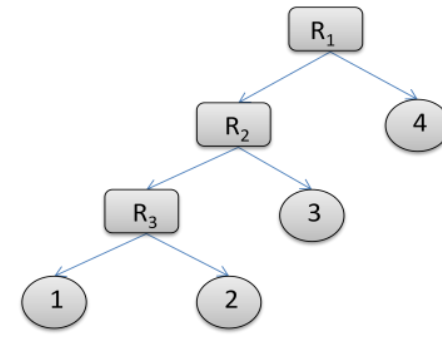

(a)

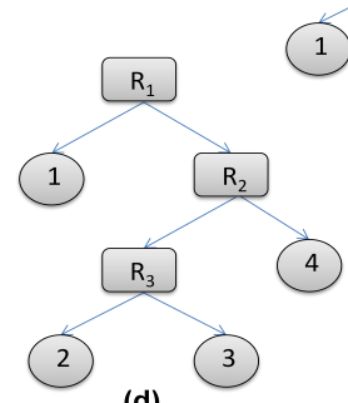

(d)

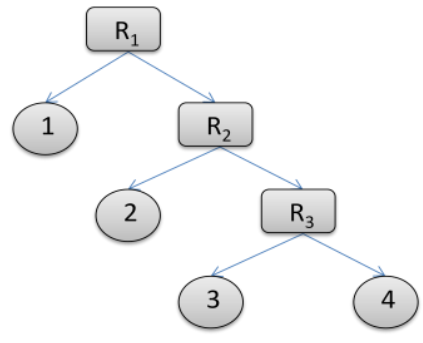

(c)

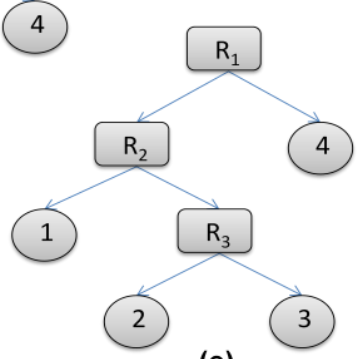

(e)

Fig. 2. Different representations as binary trees for a text with 4 edus

\subsection{The Nuclearity Score}

As known, in both RST and VT, the nuclearity is very important. Based on OS, we can develop a Nuclearity Score (NS). This will take into consideration, besides the overlapping spans under nodes, as given by the OS, the nuclearity of the relations for the corresponding overlapping tuples.

NS is calculated by multiplying OS with the ratio between the number of overlapping tuples with identical nuclearity (let's call these nuclearity-overlapping tuples) and the total number of overlapping tuples.

$$
\mathrm{NS}=\mathrm{OS} * \frac{\# \text { nuclearity-overlapping tuples }}{\text { \#overlaping tuples }} .
$$

After applying formula (2) in (3) and simplification with \#overlapping tuples, formula (4) is obtained:

$$
\mathrm{NS}=\frac{\# \text { nuclearity-overlapping tuples }}{\text { total } \# \text { tuples }} .
$$

The difference between Fig. 2 and Fig. 3 is that the last one includes also marking for the nuclearity of the parrent nodes. To exemplify how NS is computed, let's consider the discourse trees of Fig. 3(b) and Fig. 3(c): there are two pairs of overlapping 
tuples: (N_S[1,2,4] - N_S $[1,1,4])$ and (N_S[3,3,4] - S_N $[3,3,4]$ ), out of which only one pair is also a nuclearity-overlapping tuple: (N_S[1,2,4] - N_S $[1,1,4])$. Thus, formula (4) will give NS $=1 / 3$.

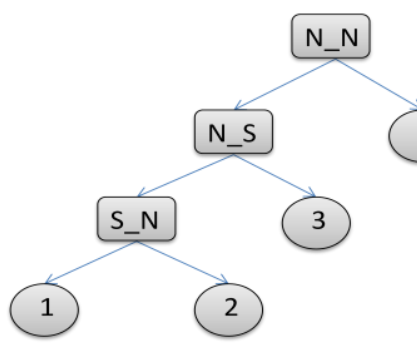

(a)
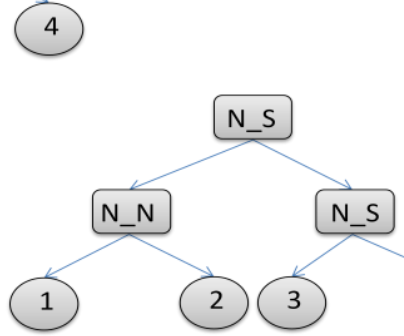

\section{$\mathrm{N} \_\mathrm{N}$}

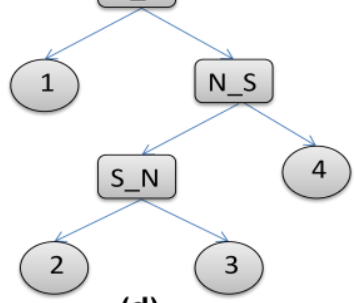

(d)

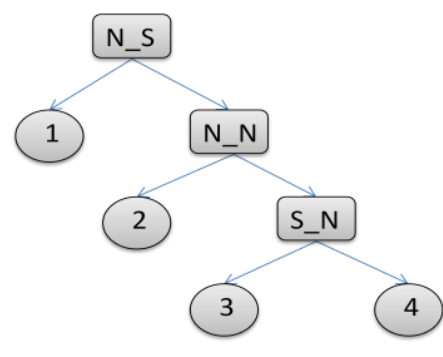

(c)

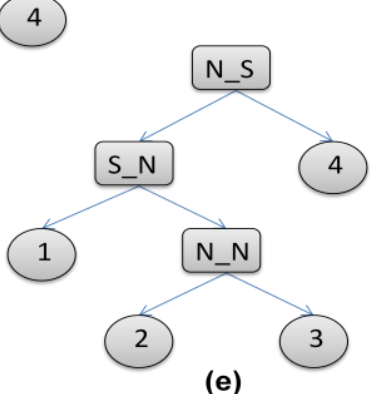

Fig. 3. Examples of tree representations including nuclearity markings

Like in the previous case, if the score is 1 , it means that both trees are identically in terms of the topology but also the nuclearity. The way the two scores have been defined makes that the Nuclearity Score be less then or equal with the Overlapping Score for the same pair of trees, showing that NS is more restrictive than OS. When NS is significantly lower than OS, this will highlight differences in nuclearity. Let's note also that nuclearity is relevant only for sub-structures that overlap.

An indicator like NS is important in applications like summarization, where nuclei are relevant. As an observation, considering the fact that the scores depend on each other, this means that if OS is nil then NS will be also nil.

\subsection{The Vein Scores}

The third proposal we make for comparing discourse structures takes into consideration Precisions, Recall and F-measures on vein expressions. Vein expressions can be computed only on discourse trees that display the nuclearity of nodes. As shown in Sections 2 and 3 above this can be done on both RST and VT annotated trees and the computation proceeds as was presented in Section 3 above. VT uses the vein expressions to measure the coherence of discourse segments as well as of the global discourse [8]. As such, Vein Scores, in addition to the other two scores defined earlier, 
place centrally the possibility to compare discourses on the ground of coherence, in the measure in which this is implicitly incorporated in tree structure. The formulas are as follows:

$$
\begin{gathered}
\text { VS-Precision }=\frac{\sum_{i=1}^{N} \frac{\mathrm{IL}}{T_{i}}}{N} . \\
\text { VS-Recall }=\frac{\sum_{i=1}^{N} \frac{\mathrm{IL}_{\mathrm{i}}}{\mathrm{G}_{\mathrm{i}}}}{N} . \\
\text { VS-FMeasure }=\frac{2 * \text { VS-Precision } * \text { VS-Recall }}{\text { VS-Precision }+ \text { VS-Recall }} .
\end{gathered}
$$

$\mathrm{IL}_{\mathrm{i}}$ - represents the number of identical labels from the vein expression of the $e d u i$ in the test tree and the gold tree;

$\mathrm{T}_{\mathrm{i}}$ - represents the total number of labels of the vein expression for $e d u i$ in the test tree;

$\mathrm{G}_{\mathrm{i}}$ - represents the total number of labels of the vein expression for $e d u i$ in the gold tree;

$\mathrm{N}$ - represents the total number of $e d u \mathrm{~s}$.

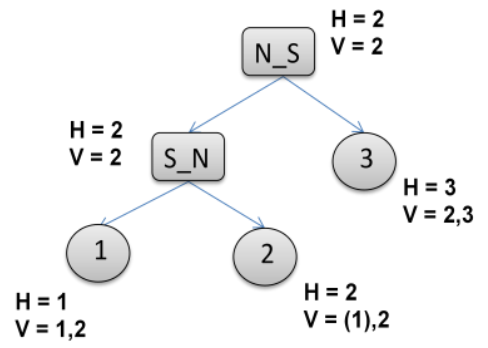

(a)

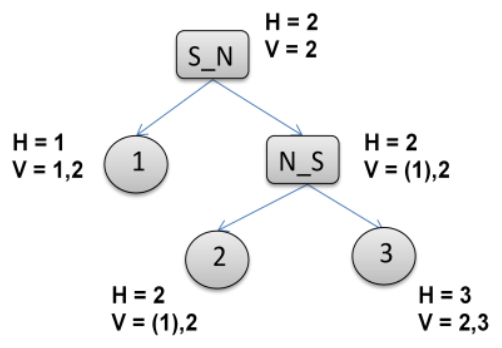

(b)

Fig. 4. Two discourse tree representations including the head and vein expressions for a text with three elementary discourse units

Let us consider the two trees in Fig. 4, with three edus in which the head $(\mathrm{H})$ and vein (V) expressions are noted. Suppose that the discourse tree of Fig. 4(a) is a test tree and the tree of Fig. 4(b) is the gold tree. Applying formulas (5), (6) and (7) we obtain:

$$
\text { VS-Precision }=\frac{\sum_{i=1}^{N} \frac{I L_{\mathrm{i}}}{T_{\mathrm{i}}}}{N}=\frac{\frac{2}{2}+\frac{2}{2}+\frac{2}{2}}{3}=\frac{3}{3}=1
$$




$$
\begin{gathered}
\text { VS-Recall }=\frac{\sum_{i=1}^{N} \frac{I L_{\mathrm{i}}}{G_{\mathrm{i}}}}{N}=\frac{\frac{2}{2}+\frac{2}{2}+\frac{2}{2}}{3}=\frac{3}{3}=1 \\
\text { VS-FMeasure }=\frac{2 * \text { VS-Precision } * \text { VS-Recall }}{\text { VS-Precision }+ \text { VS-Recall }}=\frac{2 * 1 * 1}{1+1}=1
\end{gathered}
$$

Although the trees from Fig. 4 are different in terms of structure and nuclearity, it can be seen that the vein expressions of the $e d u$ s of both trees are identical, which means that they convey the same meaning.

Let us consider another example:

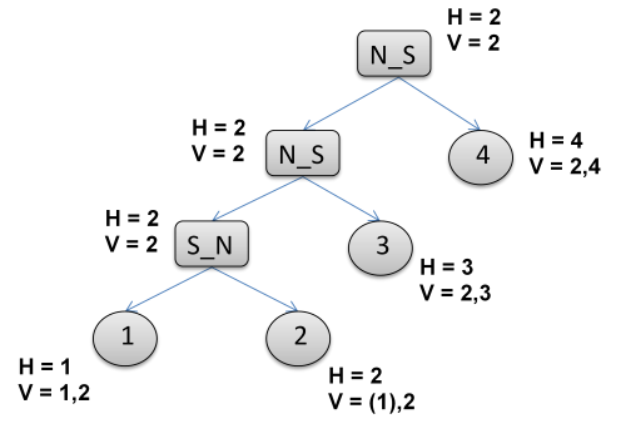

(a)

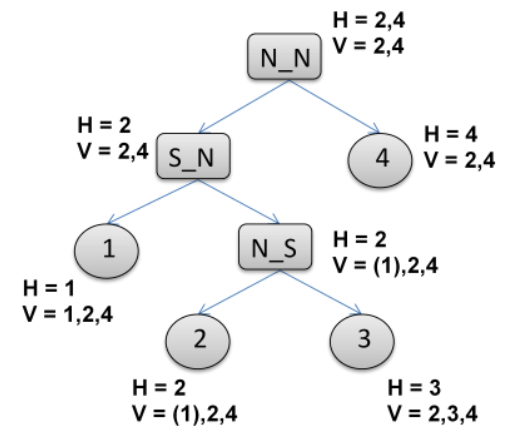

(b)

Fig. 5. Two discourse tree representations including the head and vein expressions for a text with four elementary discourse units

Applying the same formulas (5), (6) and (7) we obtain the following results:

$$
\begin{gathered}
\text { VS-Precision }=\frac{\sum_{i=1}^{N} \frac{I L_{\mathrm{i}}}{T_{\mathrm{i}}}}{N}=\frac{\frac{2}{2}+\frac{2}{2}+\frac{2}{2}+\frac{2}{2}}{4}=\frac{4}{4}=1 \\
\text { VS-Recall }=\frac{\sum_{i=1}^{N} \frac{I L_{\mathrm{i}}}{G_{\mathrm{i}}}}{N}=\frac{\frac{2}{3}+\frac{2}{3}+\frac{2}{3}+\frac{2}{2}}{4}=\frac{3}{4}=0.75 \\
\text { VS-FMeasure }=\frac{2 * \text { VS-Precision } * \text { VS-Recall }}{\text { VS-Precision + VS-Recall }}=\frac{2 * 1 * \frac{3}{4}}{1+\frac{3}{4}}=\frac{6}{7} \approx 0.857
\end{gathered}
$$

From Fig. 5 it can be seen that the two trees are totally different in terms of topology and nuclearity, but two of the $4 e d u$ s display the same vein expressions. Correspondingly, the scores computed above reflect this resemblance. 
If the first two scores are relevant for comparing discourse tree structures, this one is more relevant for summarization applications. Thus we develop a method of comparing discourse trees based on three scores beginning from the discourse structure to the summarization application, considering both Rhetorical Structure Theory and Veins Theory.

\section{Conclusion}

We have proposed three new scores for comparing discourse tree structures. The first one measures the identical coverings among all substructures of the discourse structure, the second one factors this number with identical nuclearities and the third one compares vein expressions of the terminal nodes. The idea behind these scores is to consider less distant tree structures that could manifest differences in topology if these differences are not relevant for the interpretation of the discourse. Also, our measures make possible to evaluate test structures produced by a parser even against gold structures annotated with a different theory than the one the parser relies on. Moreover, different parsers can now be evaluated and compared better, because not relevant idiosyncrasies of discourse structures pass now unnoticed by the scores.

The three measures connect well with different interests that could motivate the comparison of structures. One of the applications in which they are most useful is summarization, where the nuclearity of the units has a great importance in recognizing the main ideas.

\section{References}

1. Roark, B., Harper, M., Charniak, E., Dorr, B., Johnson, M., Kahne, J. G., Liuf, Y., Ostendorf, M., Hale, J., Krasnyanskaya, A., Lease, M., Shafran, I., Snover, M., Stewart, R., Yung, L.: SParseval: Evaluation metrics for parsing speech. In: Proceedings of LREC (2006)

2. Marcu., D.: The theory and Practice of Discourse Parsing and Summarization. MIT press (2000)

3. Soricut, R., Marcu, D.: Sentence Level Discourse Parsing using Syntactic and Lexical Information. In: Proceedings of the Human Language Technology and North American Association for Computational Linguistics Conference, pp. 149-156, Edmonton (2003)

4. Hernault, H., Prendinger, H., duVerle, D., Ishizuka, M.: HILDA: A Discourse Parser Using Support Vector Machine Classification. Dialogue and Discourse, pp. 1-33 (2010)

5. Reitter, D.: Simple signals for complex rhetorics : On rhetorical analysis with rich-features support vector models. LDV-Forum, GLDV-Journal for Computational Linguistics and Language Technology, pp. 38-52 (2003)

6. Baldridge, J., Lascarides, A.: Probabilistic head-driven parsing for discourse structure. In : Proceedings of the Ninth Conference on Computational Natural Language Learning, pp. 96103. Ann Arbor, Michigan (2005)

7. Mann, W.C., Thompson, S.A.: Rhetorical Structure Theory: Toward a functional theory of text organization. Text, pp.243-281 (1988) 
8. Taboada, M., Mann, W.C.: Rhetorical Structure Theory: looking back and moving ahead. Discourse Studies, pp. $423-459$ (2006)

9. Cristea, D., Ide, N., Romary, L.: Veins theory: A model of global discourse cohesion and coherence. In: Proceedings of the 17th international conference on Computational linguistics, pp. 281-285, Montreal (1998)

10. Davis, T.: Catalan Numbers, http://www.geometer.org/mathcircles 